\title{
Fatores associados ao conhecimento de locais públicos de esporte e lazer nas capitais brasileiras
}

\author{
Factors associated with knowledge of public places of sport and leisure in Brazilian \\ capitals
}

\section{AUTORES \\ Paula Agrizzi ${ }^{1}$ (D) \\ Thalia Eloisa Pereira Sousa Dourado ${ }^{1}$ Juliana Ilídio da Silva ${ }^{2}$ (D) \\ Amanda Cristina de Souza Andrade 2 (D) \\ 1 Universidade Federal de Mato Grosso, Faculdade de Nutrição, Cuiabá, Mato Grosso, Brasil. \\ 2 Universidade Federal de Mato Grosso, Instituto de Saúde Coletiva, Cuiabá, Mato Grosso, Brasil.}

\section{CONTATO}

Paula Agrizzi paulaagrizzi@gmail.com

Rua Quarenta e Nove, n. 2367, Boa Esperança, Cuiabá, Mato Grosso, Brasil. CEP: 78060-900.

DOI

$10.12820 /$ rbafs. $26 \mathrm{e} 0201$

\section{(cc) BY}

Este trabalho está licenciado com uma Licença Creative Commons - Atribuição 4.0 Internacional.

\begin{abstract}
RESUMO
O objetivo foi avaliar o conhecimento sobre locais públicos de esporte e lazer nas capitais brasileiras e sua relação com fatores sociodemográficos e do ambiente urbano. Estudo com dados da Pesquisa Nacional de Saúde, edição no ano de 2013, inquérito de base domiciliar, representativo da população adulta residente em todas as capitais do Brasil. A variável de desfecho foi conhecimento sobre locais públicos de esporte e lazer. As variáveis de exposição foram sexo, faixa etária, escolaridade e Índice de Bem-Estar Urbano (IBEU), que avalia as condições coletivas de vida na cidade por meio dos dados do Censo Demográfico de 2010. Realizou-se análise bivariada através do teste Qui-quadrado e correlação de Pearson. Dos 27.017 entrevistados, 56,6\% (IC95\%: 55,1 - 58,1) relataram conhecer locais públicos de esporte e lazer, sendo maior entre homens ( $58,2 \%$ vs $55,3 \%$ ) e mais escolarizados $(45,5 \%$ de 0 a 4; 49,2\% de 5 a 8; 55,2\% de 9 a 11; 68,2\% de 12 anos ou mais). Não foi observada diferença entre as faixas etárias. Foi observada correlação positiva entre proporção de conhecimento e IBEU $(\mathrm{r}=0,709 ; \mathrm{p}<0,05)$. As capitais com melhores indicadores de conhecimento e do ambiente urbano foram Vitória, Brasília, Goiânia e Curitiba. Diante disso, a melhoria e criação de espaços públicos de lazer deve considerar as desigualdades na sua distribuição em relação ao ambiente e as características sociodemográficas da população, para contribuir para a promoção da atividade física e, consequentemente, para melhorar a saúde e equidade social.
\end{abstract}

Palavras-chave: Saúde urbana; Inquérito; Desigualdades; Locais públicos de lazer.

\section{ABSTRACT}

The objective was to evaluate the knowledge about public places of sport and leisure in the Brazilian capitals and their relationship with sociodemographic factors and the urban environment. Study with data from the National Health Survey, edition in the year 2013, a household-based survey, representative of the adult population residing in all capitals of Brazil. The outcome variable was knowledge about public places of sport and leisure. The exposure variables were sex, age group, education and Urban Well-Being Index (IBEU), which assesses the collective living conditions in the city troughthout data from the 2010 demographic census. A bivariate analysis was performed using the chi-square test and Pearson's correlation. Of the 27,017 interviewees, 56.6\% (95\% CI: 55.1 - 58.1) reported knowing public places of sport and leisure, being higher among men (58.2\% vs 55.3\%) and more educated (45.5\% from 0 to 4; $49.2 \%$ from 5 to 8; $55.2 \%$ from 9 to $11 ; 68.2 \%$ from 12 years or older). There was no difference between the age groups. A positive correlation was observed between the proportion of knowledge and IBEU $(r=0.709 ; p<0.05)$. The capitals with the best knowledge and urban environment indicators were Vitória, Brasilia, Goiânia and Curitiba. Therefore, the improvement and creation of public leisure spaces must take account into the inequalities distribution in relation to the environment and the sociodemographic characteristics of the population, to contribute to the promotion of physical activity and, consequently, to improve health and social equity.

Keywords: Urban health; Surveys and questionnaires; Socioeconomic factors; Sports and recreational facilities.

\section{Introdução}

A disponibilidade de espaços públicos de lazer em áreas urbanas contribui para a promoção de um estilo de vida ativo e melhora a qualidade de vida e opções de lazer da população ${ }^{1,2}$. Além disso, associa-se também a benefícios sociais, como aumento da participação e convívio social, econômicos, com maior oferta de ser- viços, e benefícios ambientais com a reformulação do espaço urbano e preservação de áreas verdes ${ }^{3}$.

Os estudos sobre a temática têm avaliado a presença de locais públicos de lazer à uma distância próxima da residência ${ }^{4}$, os tipos de estruturas existentes ${ }^{5,6}$, características do ambiente construído no entorno desses locais $^{3}$, a disponibilidade de equipamentos ${ }^{3,7,6}$, a qualidade 
dos espaços ${ }^{8}$ ou a percepção em relação ao uso ${ }^{9,10}$, como segurança, acessibilidade e condições das estruturas.

Os espaços públicos de lazer como parques, praças e ciclovias são locais que oferecem condições de segurança e são gratuitos e acessíveis ${ }^{11}$. Nesse sentido, os espaços públicos tradicionais das cidades podem influenciar na vida ativa da populaçãa $0^{1,12,13}$. Entretanto, o uso desses locais pelos indivíduos sofre a influência de variáveis, tais como: idade, sexo, nível econômico, localização e distribuição dentro da cidade, estrutura apresentada, condições de limpeza, manutenção, estética e trânsito ${ }^{7,9,6}$.

Um estudo realizado com dados de 11 países, incluindo o Brasil, mostrou diferenças significativas na disponibilidade de espaços públicos de lazer entre diferentes países, sendo menos frequente nos países de renda baixa e média. No Brasil, somente 28,3\% dos entrevistados relataram a presença de instalações públicas de lazer ou de baixo custo na sua vizinhança ${ }^{14}$.

O Brasil caracteriza-se por grande extensão territorial, marcada pelo desenvolvimento desigual entre as regiões e no interior das aglomerações urbanas ${ }^{15}$. A persistente desigualdade entre os municípios do país pode ser atribuída à segregação espacial e questões estruturais como a distribuição do sistema de transporte e da infraestrutura, e do desenvolvimento urbano ${ }^{16,17}$.

As condições de acesso e as oportunidades de atividades de lazer são desigualmente distribuídas no território e entre grupos sociais ${ }^{7,9,18-20}$. Os estudos realizados no Brasil sobre a temática se limitam a cidades específi$\mathrm{cas}^{7}$, contrapondo a proposta aqui abordada, que busca identificar os diferenciais socioespaciais existentes no território brasileiro. Assim, o objetivo deste estudo foi avaliar o conhecimento sobre locais públicos de esporte e lazer nas capitais brasileiras e sua relação com fatores sociodemográficos e do ambiente urbano.

\section{Métodos}

Estudo descritivo realizado com os dados da Pesquisa Nacional de Saúde (PNS). Inquérito de base domiciliar, representativo da população adulta residente em todas as regiões do Brasil, e que teve sua primeira edição no ano de 2013. O processo amostral foi realizado por conglomerados em três estágios: setores censitários; domicílio e um morador de 18 anos ou mais. Em cada domicílio foi selecionado de forma aleatória um morador adulto, o qual foi convidado a participar da pesquisa e a responder um questionário sobre estilo de vida e saúde, características do domicílio e sobre os outros moradores. Foram realizadas 60.202 entrevistas com um percentual de perdas de 25,9\%. A PNS tem como níveis de desagregação as grandes regiões nacionais, unidades da federação, capitais e regiões metropolitanas. Maiores informações estão disponíveis em publicação prévia ${ }^{21}$. No presente estudo foram incluídos somente os adultos residentes nas 26 capitais e Distrito Federal ( $\mathrm{n}=27.017$ ), por ser tratar da menor unidade de desagregação geográfica disponível.

Para o estudo foi adotada duas abordagens analíticas, a primeira, um estudo transversal, e a segunda, um estudo ecológico utilizando medidas agregadas do primeiro estudo e indicadores do ambiente urbano obtidos a partir de dados do Censo Demográfico.

Para a abordagem transversal a variável de desfecho foi o conhecimento sobre locais públicos de esporte e lazer medida pela pergunta: "Perto do seu domicílio, existe algum lugar público (praça, parque, rua fechada e praia) para fazer caminhada, realizar exercício ou praticar esporte?" (sim e não). As variáveis de exposição foram: sexo (feminino e masculino), idade em faixa etária (18-34, 35-59 e 60 ou mais), escolaridade em anos de estudo (0-4, 5-8, 9-11 e 10 ou mais).

Para a abordagem ecológica a variável de desfecho foi a proporção de conhecimento sobre locais públicos de esporte e lazer, obtida a partir da agregação por capital da variável conhecimento originalmente medida em nível individual. A variável de exposição foi o Índice de Bem-Estar Urbano (IBEU) e suas dimensões, das 26 capitais e Distrito Federal.

O IBEU foi construído para todos os municípios brasileiros, utilizando informações do Censo Demográfico de 2010. Esse índice permite avaliar a dimensão urbana do bem-estar usufruído pelos cidadãos quanto aos serviços sociais prestados pelo Estado, relacionando com as condições coletivas de vida na cidade, nas escalas da habitação e da vizinhança próxima, e pelos equipamentos e serviços urbanos. O IBEU-Municipal foi obtido pela média aritmética de suas cinco dimensões: Mobilidade Urbana (D1), Condições Ambientais Urbanas (D2), Condições Habitacionais Urbanas (D3), Atendimento de Serviços Coletivos Urbanos (D4) e Infraestrutura Urbana (D5). O índice varia entre zero (piores condições) e um (melhores condições de bem- estar urbano), e pode ser classificado em ruim ou muito ruim (menor que 0,7 ), médio (entre 0,7 e 0,8 ) e bom ou muito bom (maior que 0,8$)^{17}$.

A dimensão de mobilidade urbana (D1) utiliza o indicador de deslocamento casa-trabalho, que consiste no tempo de deslocamento que as pessoas que trabalham fora, usam no trajeto de ida entre o domicílio 
em que reside e o local de trabalho. A dimensão de condições ambientais urbanas (D2) foi estabelecida utilizando três indicadores: arborização, esgoto a céu aberto e lixo acumulado do entorno dos domicílios. A dimensão de condições habitacionais urbanas (D3) foi estabelecida utilizando cinco indicadores: aglomerado subnormal, densidade domiciliar, densidade morador/ banheiro, material das paredes dos domicílios e espécie do domicílio ${ }^{17}$. A dimensão de atendimento de serviços coletivos urbanos (D4) foi criada a partir de quatro indicadores: atendimento adequado de água, atendimento adequado de esgoto, atendimento adequado de energia e coleta adequada de lixo, expressando serviços públicos essenciais para o bem-estar urbano. A dimensão de infraestrutura urbana (D5) foi estabelecida por sete indicadores: iluminação pública, pavimentação, calçada, meio-fio/guia, bueiro ou boca de lobo, rampa para cadeirantes e logradouros, que representam as condições que podem melhorar a qualidade de vida da população ${ }^{17}$. Para maiores informações, os dados do IBEU estão disponíveis na página de Internet do Observatório das Metrópoles ${ }^{22}$.

$\mathrm{Na}$ análise transversal foram calculadas frequências absolutas e relativas, e aplicou-se o teste Qui-quadrado de Pearson para avaliar a associação entre o conhecimento sobre locais públicos de esporte e lazer (sim; não) e as variáveis sexo, faixa etária e escolaridade. $\mathrm{Na}$ análise ecológica, primeiro foi verificado a normalidade da distribuição das variáveis proporção de conhecimento, IBEU e suas dimensões pelo teste de Shapiro-Wilk, e em seguida, foi calculado o coeficiente de correlação de Pearson ( $\mathrm{r}$ ). Quanto mais próximo de +1 , mais forte é a correlação positiva, quanto mais próximo de -1 mais forte é a correlação negativa e quando $r=0$, não existe associação linear. A correlação foi classificada como fraca $(r<0,3)$, moderada $(0,3 \leq r<0,6)$ e forte $(r \geq 0,6)^{23}$. Foi adotado um nível de significância de $5 \%$ e as análises foram realizadas no software STATA versão 12.0.

O estudo foi aprovado pela Comissão Nacional de Ética em Pesquisa (CONEP), sob o Parecer no 328.159, de 26 de junho de 2013. Todos os indivíduos participantes assinaram um Termo de Consentimento Livre e Esclarecido.

\section{Resultados}

Dos 27.017 entrevistados, 54,9\% eram do sexo feminino, $44,2 \%$ tinham entre 35 e 59 anos e $38,1 \%$ possuíam de 9 a 11 anos de estudo. No conjunto das capitais e Distrito Federal, o conhecimento de locais públicos de es- porte e lazer foi relatado por 56,6\% (IC95\%: 55,1 - 58,1) dos entrevistados, sendo maior entre homens $(58,2 \%$ vs $55,3 \%)$ e mais escolarizados ( $45,5 \%$ de 0 a $4 ; 49,2 \%$ de 5 a $8 ; 55,2 \%$ de 9 a $11 ; 68,2 \%$ de 12 anos ou mais). Não foi observada diferença entre as faixas etárias (Tabela 1 ).

Tabela 1 - Conhecimento de locais públicos de esporte e lazer conforme variáveis sociodemográficas. Pesquisa Nacional de Saúde, Brasil, 2013 ( $\mathrm{n}=27.017)$.

\begin{tabular}{lccc}
\hline \multirow{2}{*}{ Variáveis } & Total & $\begin{array}{c}\text { Conhecimento de } \\
\text { locais públicos de } \\
\text { esporte e lazer }\end{array}$ & \multirow{2}{*}{ Valor $\mathrm{p}^{1}$} \\
\cline { 2 - 3 } & $\%(\mathrm{IC} 95 \%)$ & \% (IC 95\%) & \\
\cline { 2 - 3 } Sexo & & & \\
Feminino & $54,9(44,2 ; 45,9)$ & $55,3(53,6 ; 57,0)$ & 0,002 \\
Masculino & $45,0(54,0 ; 55,9)$ & $58,2(56,3 ; 59,9)$ & \\
Faixa etária & & & \\
18-34 & $37,7(36,8 ; 38,6)$ & $57,1(55,1 ; 59,1)$ & 0,701 \\
35-59 & $44,2(43,3 ; 45,1)$ & $56,2(54,4 ; 58,0)$ & \\
60 ou mais & $18,0(17,3 ; 18,9)$ & $56,6(54,1 ; 59,0)$ & \\
Escolaridade & & & \\
0-4 anos & $12,3(11,7 ; 13,0)$ & $45,5(42,7 ; 48,3)$ & \\
5-8 anos & $20,2(19,3 ; 21,0)$ & $49,2(46,6 ; 51,7)$ & \\
9-11 anos & $38,1(37,1 ; 39,1)$ & $55,2(53,2 ; 57,2)$ & $<0,001$ \\
12 ou mais & $29,4(28,2 ; 30,6)$ & $68,2(66,2 ; 70,2)$ & \\
\hline
\end{tabular}

IC95\% = Intervalo de 95\% de confiança; ${ }^{1}$ Teste Qui-quadrado de Pearson.

As capitais apresentam distribuição heterogênea em relação às Condições de Bem-Estar Urbano (Figura 1A). Doze capitais apresentam Índices de Bem-Estar Urbano maior que 0,8 (Vitória, Goiânia, Curitiba, Belo Horizonte, Porto Alegre, Campo Grande, Aracaju, Natal, Florianópolis, Palmas, Brasília e São Paulo) e cinco com índices menores que 0,7 , sendo todas localizadas na região Norte (Rio Branco, Manaus, Belém, Porto Velho e Macapá).

As capitais com proporções de conhecimento de locais públicos de esporte e lazer maiores que $70 \%$ foram Vitória (79,3\%), Brasília (74,4\%), Goiânia (73,9\%) e Curitiba $(70,4 \%)$ e com menos de $40 \%$ foram Porto Velho (25,9\%), São Luís (31,6\%), Rio Branco (33,1\%) e Cuiabá $(37,9 \%)$, como mostra a Figura 1B.

Correlações positivas e moderada a forte foram observadas para as dimensões condições ambientais urbanas $(r=0,608 ; p<0,05)$, condições habitacionais urbanas $(\mathrm{r}=0,641 ; \mathrm{p}<0,05)$, atendimento de serviços coletivos urbanos $(r=0,522 ; \mathrm{p}<0,05)$ e infraestrutura urbana $(\mathrm{r}=$ 0,644; $p<0,05)$, com exceção da dimensão mobilidade urbana $(r=0,008 ; p>0,05)$ que não apresentou correlação significativa, como mostra a Figura 2. Foi observada 


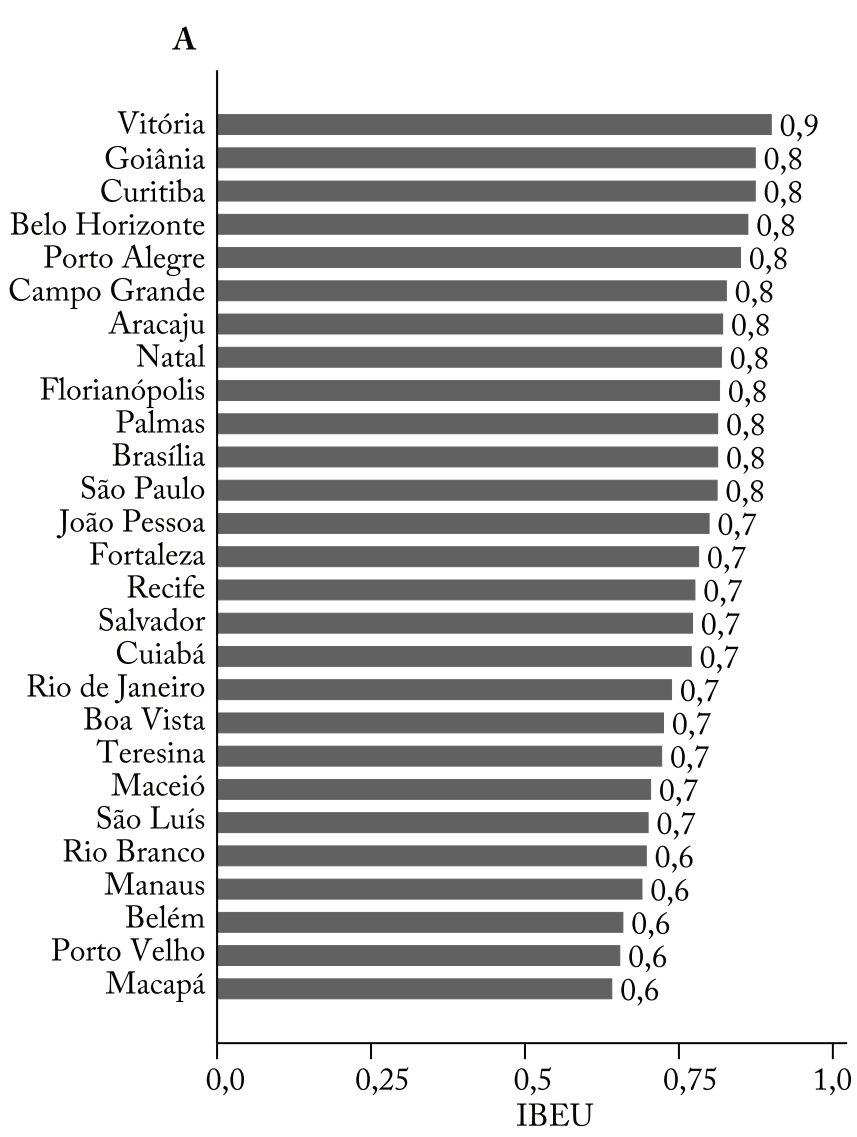

Figura 1 - Proporção de conhecimento de locais públicos de lazer (a) e IBEU (b) das capitais e Distrito Federal. Pesquisa Nacional de Saúde, 2013.

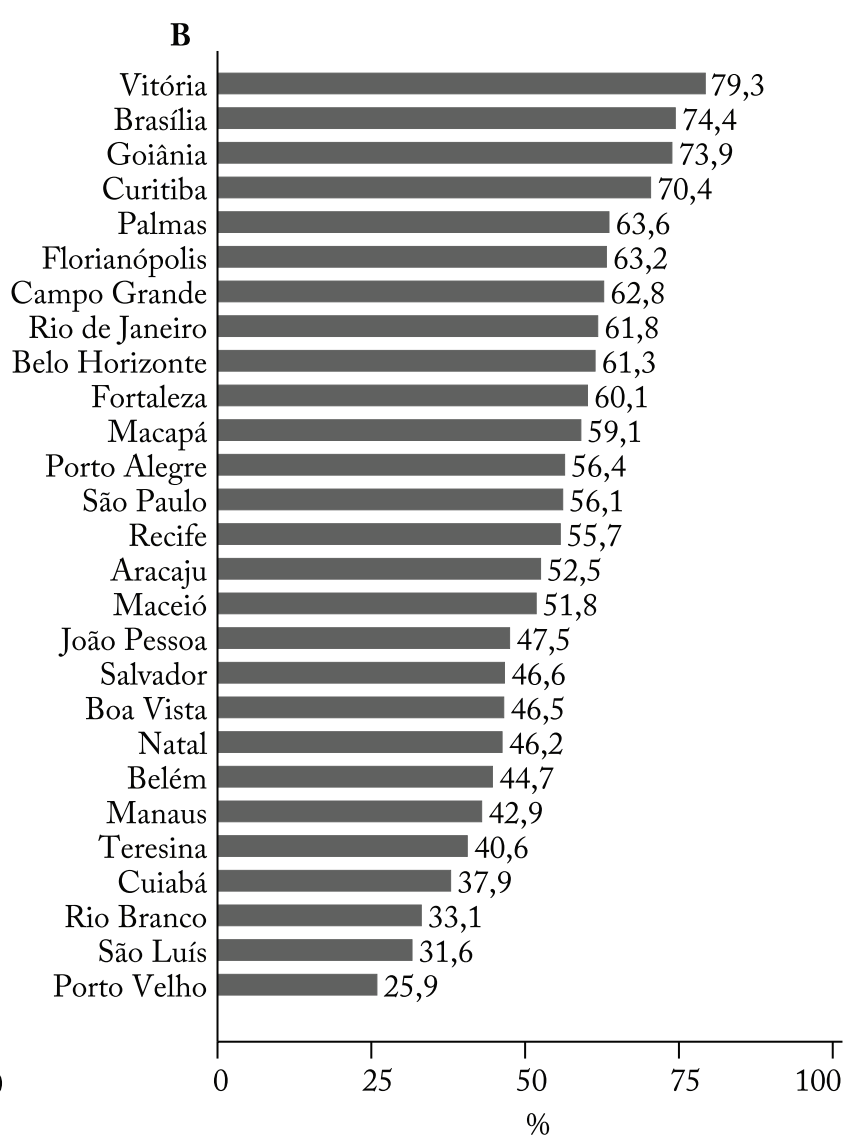

\section{B}

Vitória

Goiânia

Curitiba

Palmas

Campo Grande

Rio de Janeiro

Fortaleza

São Paulo

Recife

Aracaju

Salvador

Boa Vista

Manaus

Teresina

io Branco

São Luís

Porto Velho correlação positiva e forte entre a proporção de conhecimento de locais públicos de esporte e lazer e o IBEU ( $\mathrm{r}$ = 0,709; $\mathrm{p}<0,05)$, destacando-se as capitais de Vitória, Goiânia e Curitiba que apresentaram maior proporção de conhecimento e índice do IBEU (Figura 3).

\section{Discussão}

No presente estudo, o conhecimento de locais públicos de esporte e lazer nas capitais brasileiras foi mais frequente entre homens, mais escolarizados, e sem diferença entre as faixas etárias. O maior conhecimento desses locais foi observado nas capitais com melhores atributos do ambiente, avaliados pelo IBEU e suas dimensões, condições ambientais urbanas, condições habitacionais urbanas, atendimento de serviços coletivos urbanos e infraestrutura urbana.

No conjunto das 26 capitais e Distrito Federal, 56\% dos entrevistados relataram ter conhecimento sobre os locais públicos de esporte e lazer e as cidades que apresentaram maior proporção foram Vitória, Brasília, Goiânia e Curitiba.Um estudo realizado com residentes nas regiões metropolitanas de São Paulo, Rio de Janeiro, Porto Alegre, Salvador, Manaus e Brasília identificou que $81 \%$ da população mencionou conhecer pelo menos um parque urbano, tendo sido listados 171 nomes. Isso pode estar relacionado à localização desses parques, normalmente nos centros urbanos ${ }^{24}$. A diferença observada na proporção de conhecimento de locais públicos de esporte e lazer entre os estudos poderia ser atribuída a diferença na forma de mensuração da variável, no processo amostral e composição das amostras.

Sakata \& Gonçalves ${ }^{25}$ em estudo realizado com conjunto de 13 capitais brasileiras e o Distrito Federal, entre 2000 e 2017, identificaram que o número de parques urbanos no Brasil dobrou, com surgimento de 240 novos parques no período. A expansão de parques nas cidades pode ser atribuída a mudanças na legislação ambiental, como meio de preservação de recursos naturais e provisão de espaços de lazer e esporte ${ }^{25}$. Esse processo não foi homogêneo entre as cidades, sendo menos frequente no Rio de Janeiro, Maceió, Fortaleza e Florianópolis, nas quais as orlas ainda são os grandes parques urbanos ${ }^{25}$. Enquanto Vitória, Recife, São Paulo, Belo Horizonte, Brasília, Campo Grande e Curitiba, são exceções, que investiram na criação de parques novos ${ }^{25}$. 


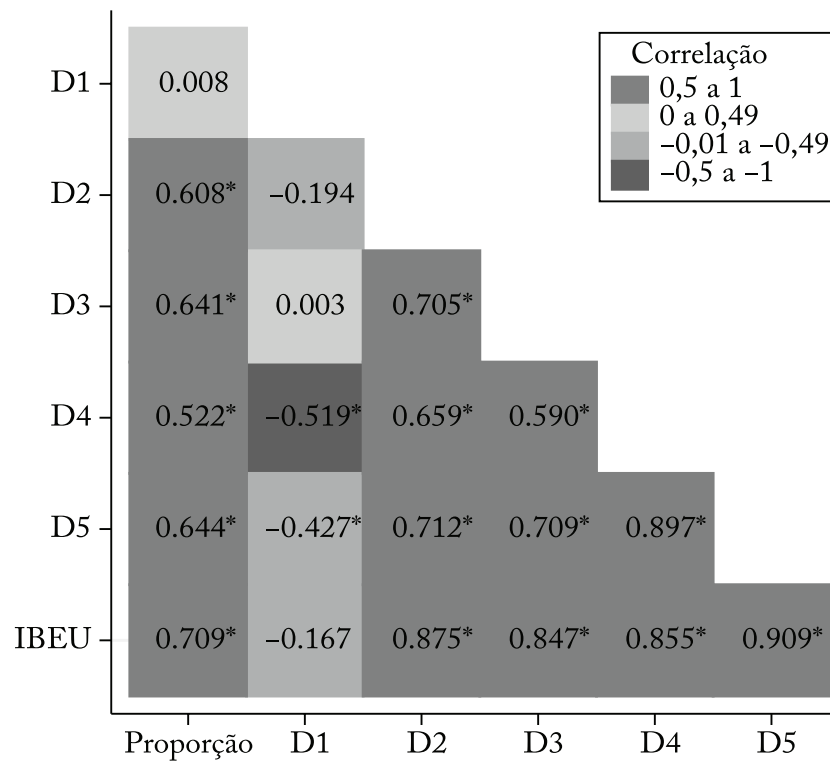

Figura 2 - Matriz de correlação entre proporção de conhecimento de locais públicos de esporte e lazer e IBEU e seus domínios das capitais e Distrito Federal. Pesquisa Nacional de Saúde, 2013. * $\mathrm{p}<0,05$; Proporção = proporção de conhecimento de locais públicos de esporte e lazer; IBEU = Índice de Bem-estar Urbano; D1 = Dimensão 1 (Mobilidade urbana); D2 = Dimensão 2 (Condições Ambientais Urbanas), D3 = Dimensão 3 (Condições Habitacionais Urbanas); D4 = Dimensão 4 (Atendimento de Serviços Coletivos Urbanos); D5 = Dimensão 5 (Infraestrutura Urbana).

Segundo Fermino \& Reis ${ }^{7}$, em uma revisão sistemática sobre o uso de espaços públicos abertos de lazer, definidos como locais com estruturas capazes de atrair a população e facilitar a realização de atividades físicas, o equipamento mais frequentemente investigado nos estudos foram os parques. Os autores verificaram que poucos estudos avaliaram outros tipos de espaços como pistas de caminhada, centros recreativos, entre outros. O uso desses locais tem sido associado a características individuais, como sexo masculino, idade mais jovem, ser ativo fisicamente, e ambientais, como a proximidade da residência.

No presente estudo a frequência de conhecimento de locais públicos de esporte foi maior entre homens e indivíduos de maior escolaridade, e não diferiu entre as faixas etárias para o conjunto das capitais e Distrito Federal. A comparação dos resultados foi dificultada pela ausência na literatura de estudos que avaliassem o conhecimento de locais de esporte e lazer. Os estudos existentes têm investigado a associação entre fatores sociodemográficos e o uso de locais de esporte e lazer $^{4,7,26}$ e a prática de atividade física ${ }^{13,27}$.

Fermino et al. $^{4}$, que investigaram os fatores individuais e ambientais associados ao uso de parques e praças por adultos em Curitiba não encontraram associa-

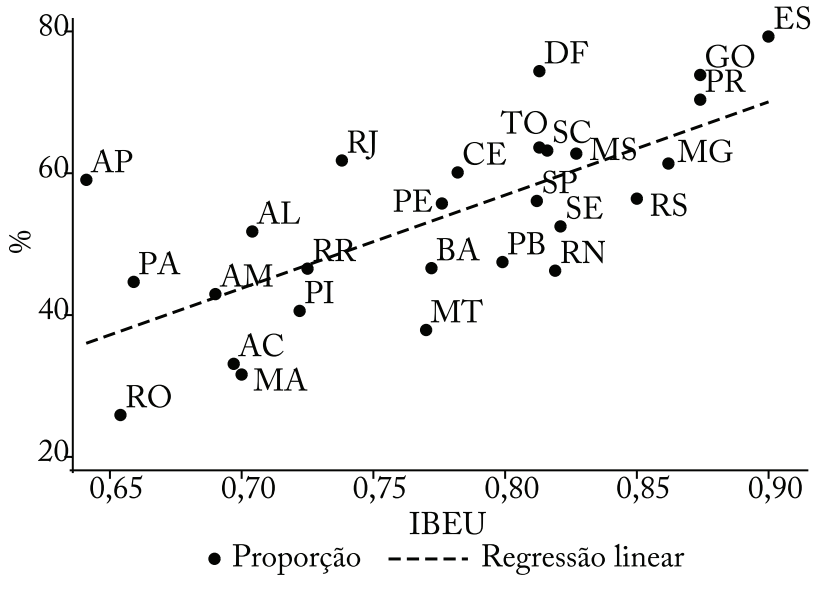

Figura 3 - Análise de correlação entre IBEU e proporção de conhecimento de locais públicos de esporte e lazer das capitais e Distrito Federal. Pesquisa Nacional de Saúde, 2013.

* Proporção = proporção de conhecimento de locais públicos de esporte e lazer; IBEU = Índice de Bem-estar Urbano; Como as capitais brasileiras não possuem suas próprias siglas foi utilizado no gráfico as siglas de seus respectivos estados: $\mathrm{AC}=\mathrm{Rio}$ Branco; $\mathrm{AL}=$ Maceió; $\mathrm{AP}=$ Macapá; $\mathrm{AM}=$ Manaus; $\mathrm{BA}=$ Salvador; $\mathrm{CE}=$ Fortaleza; $\mathrm{DF}=$ Brasília; $\mathrm{E}$ S= Vitória; $\mathrm{GO}=$ Goiânia; $\mathrm{MA}=$ São Luís; $\mathrm{MT}=$ Cuiabá; $\mathrm{MS}$ = Campo Grande; $\mathrm{MG}=$ Belo Horizonte; $\mathrm{PA}=$ Belém; $\mathrm{PB}=$ João Pessoa; $\mathrm{PR}=$ Curitiba PE = Recife P I= Teresina; RJ = Rio de Janeiro; RN = Natal; RS = Porto Alegre; $\mathrm{RO}$ = Porto Velho; RR = Boa Vista; $\mathrm{SC}=$ Florianópolis; $\mathrm{SP}$ = São Paulo; $\mathrm{SE}=$ Aracaju; $\mathrm{TO}=$ Palmas .

ção com faixa etária. Entretanto, o estudo de Fermino \& Reis $^{7}$, em revisão sistemática sobre o uso de locais públicos de esporte e lazer identificou maior frequência de uso desses locais entre os mais jovens.

Fermino et al. ${ }^{26}$ que analisou a associação entre a frequência de uso de parques e a prática de atividade física em residentes de Curitiba identificaram que essa relação possui efeitos mais consistentes entre os homens. Essa diferença entre os sexos pode estar relacionada a fatores culturais relativos à criação, com maior incentivo de atividades ao ar livre para homens e menor para as mulheres. Também pode ser atribuída à dupla jornada de trabalho entre as mulheres que inclui tarefas domésticas e trabalho remunerado, propiciando a estas menor tempo para outras atividades ${ }^{28}$, como a prática de atividade física e a realização de atividades de lazer.

Malta et al. ${ }^{27}$, utilizando os dados do VIGITEL referente a população adulta das capitais brasileiras e Distrito Federal entre 2006 e 2013, apontaram que a prática de atividade física no tempo livre foi mais frequente entre os homens, jovens e mais escolarizados.

Boclin et al. ${ }^{13}$ analisaram a associação entre características da vizinhança e a prática de atividade física no lazer, no município do Rio de Janeiro e identificaram 
maior prevalência entre indivíduos com nível superior completo, renda familiar per capita maior que seis salários mínimos e residentes em bairros com maiores índices de desenvolvimento social. Os grupos populacionais socialmente mais vulneráveis residem em áreas de desvantagens ambientais, sociais e econômicas e que se encontram concentradas, evidenciando-se as desigualdades espaciais dentro das cidades ${ }^{20}$.

Foi observada correlação positiva entre conhecimento de locais públicos de esporte e lazer e condições do ambiente urbano, sendo ambos indicadores maiores para Vitória, Goiânia e Curitiba. Esses três municípios destacam-se pelas ações adotadas nos últimos anos para criação de parques em diferentes áreas da cidade. A cidade de Curitiba é reconhecida por adotar estratégias de planejamento urbano conciliadas com a criação de parques e áreas verdes. Goiânia teve um aumento expressivo nas áreas de parque na cidade, até 1996 havia somente três parques implantados e, em 2016, eram 39. Vitória tem sido pioneira na institucionalização da proteção ambiental e no uso de recursos dos termos de compensação ambiental ou de ajustamento de conduta para criação e manutenção de parques ${ }^{25}$.

O IBEU e suas dimensões permitem a partir da avaliação das características essenciais do espaço urbano conhecer as desigualdades socioespaciais existentes entre as capitais brasileiras. As condições urbanas foram piores nas capitais localizadas nas regiões Norte do país e as melhores nas regiões Sudeste e Sul, sendo a Região Centro-Oeste um espaço de transição das condições de bem-estar urbano ${ }^{17}$.

Conforme relatório do IBEU municipal ${ }^{17}$, que avaliou o índice de bem-estar urbano dos municípios brasileiros, a dimensão de mobilidade urbana apresentou maior variabilidade entre as regiões, e foi pior apenas no Rio de Janeiro e São Paulo, capitais mais populosas. As condições ambientais e habitacionais urbanas foram favoráveis na maior parte das capitais ${ }^{17}$. As capitais que apresentavam condições ruins de atendimento de serviços coletivos urbanos localizavam-se nas regiões Nordeste e Norte ${ }^{17}$. A infraestrutura urbana apresentou a pior situação de bem-estar, uma vez que somente uma capital foi classificada com nível muito bom e dezoito em condições ruins e muito ruins ${ }^{17}$.

Os espaços públicos abertos e instalações de atividade física se distribuem de forma desigual nas cidades, com menor frequência em áreas de baixo nível socioeconômico, maior densidade residencial e menor proporção de idosos ${ }^{9,13,29,30}$. Assim como os parques ur- banos, que apesar de terem uma distribuição dispersa dentro das cidades, os parques com equipamentos de maior qualidade e melhor manutenção têm sidos instalados em locais de renda mais alta ${ }^{25}$.

Entre a população residente nas regiões metropolitanas de São Paulo, Rio de Janeiro, Porto Alegre, Salvador, Manaus e Brasília, identificou-se que $67 \%$ fazia pouco ou nenhum uso de parques urbanos, sendo que a maioria desses indivíduos tinha baixo poder aquisitivo e relatou ter dificuldades para acessar esses locais devido a distância e necessidade de uso de transportes públicos, o que evidencia a concentração desses equipamentos dentro do território ${ }^{24}$.

Leite et al. ${ }^{18}$ que pesquisaram a vulnerabilidades social e em saúde e a segregação residencial no município de São Paulo, identificaram que determinados grupos sociais ocupam espaços definidos no território das cidades, de acordo com sua posição social, gerando um padrão socioespacial, que permite que um grupo tenha vantagem em detrimento de outros. Essas vantagens podem ser identificadas quando se avalia as condições dos espaços públicos de lazer nos territórios periféricos, uma vez que, estes existem em quantidades insuficientes para seus moradores e ainda apresentam precariedades e barreiras para sua utilização.

A diferença no IBEU das capitais e no conhecimento sobre locais públicos de esporte e lazer reflete a concentração da infraestrutura urbana no país e reforça os padrões de segregação espacial. O Brasil é um país de dimensão continental, com diversidade e heterogeneidade regional. Marcas do desenvolvimento desigual e do processo de urbanização, induzidas pela expansão física, com a periferização, e deslocamentos voltados à oferta de moradia, infraestrutura e serviços ${ }^{15,16}$. Os municípios em estágio mais avançado de urbanização, encontram-se nas regiões Sudeste e Sul, apresentam a maior concentração de população, do Produto Interno Bruto, do número de ocupados, de atividades não agrícolas, de mobilidade pendular da população e que apresentavam condições de vulnerabilidade social de média a baixa ${ }^{16}$.

O uso do território nas cidades é marcado pelas desigualdades sociais, onde populações mais carentes e pobres residem nas regiões mais periféricas e com piores condições de infraestrutura e serviços urbanos e de mobilida$\mathrm{de}^{18}$. Essas diferenças podem ser atribuídas ao processo de crescimento populacional e urbanização desigual, que impactam direto e indiretamente nas condições de vida e saúde da população ${ }^{19}$, e suscita a necessidade de políticas intersetoriais que visem a redução das desigualdades em 
saúde e a promoção de cidades saudáveis.

Dessa forma, a compreensão da relação entre o conhecimento sobre locais públicos de esporte e lazer perto do domicílio e as características sociodemográficas e do ambiente urbano nas capitais brasileiras é importante para subsidiar e orientar intervenções que promovam o maior acesso a estes locais e, portanto, contribuir para qualidade de vida e saúde da população. As características ambientais, especialmente a disponibilidade de espaços públicos de lazer, podem proporcionar mudança de comportamentos relacionados à saúde, contemplar grande parcela da população e diminuir as iniquidades sociais relacionadas à prática de atividade física ${ }^{12,13}$.

O presente estudo apresenta algumas limitações referentes à necessidade de averiguar a distância entre os locais públicos de esporte e lazer e as residências dos indivíduos entrevistados e investigar se os que afirmaram conhecer, de fato utilizam esses espaços. Não foi encontrado na literatura um estudo que tenha avaliado o conhecimento de locais públicos de esporte e lazer conforme mensurado no presente estudo, dificultando a comparabilidade dos resultados. Deve-se destacar ainda, que as informações selecionadas foram apenas de adultos, impedindo assim a inferência para crianças e adolescentes. Por outro lado, cabe identificar os pontos fortes deste trabalho, tais como: se tratar de um dos poucos estudos que relaciona o conhecimento de locais públicos de esporte e lazer com o IBEU de todas as capitais brasileiras e o Distrito Federal e a utilização de uma amostra nacional proveniente da PNS de 2013, que é representativa do Brasil e suas capitais ${ }^{21}$.

O estudo possibilitou identificar as características do ambiente urbano associadas ao conhecimento de locais públicos de esporte e lazer nas capitais brasileiras, para além das características individuais. Pouco mais da metade dos residentes nas capitais tinha conhecimento sobre esses locais. $\mathrm{O}$ conhecimento mostrou-se associado positivamente com indicadores de condições ambientais e habitacionais urbanas, atendimento de serviços coletivos urbanos e infraestrutura urbana, especialmente em Vitória, Goiânia e Curitiba, as quais foram as capitais com melhores indicadores.

Os achados poderão subsidiar ações que visem mitigar as desigualdades na distribuição desses locais entre as capitais. Assim, a implementação de políticas públicas para melhorar a distribuição desses locais pode aumentar o acesso a espaços propícios ao lazer e à prática de atividade física, e promover efeitos positivos sobre os níveis de atividade física e saúde da população.

\section{Conflito de interesse}

Os autores declaram não haver conflito de interesses.

\section{Contribuições dos autores}

Agrizzi P, participou da análise e interpretação dos dados; revisão da literatura; redação e revisão do artigo; aprovação final da versão do artigo a ser publicada. Dourado TEPS e Silva JI, participaram da interpretação dos dados; revisão da versão final do artigo; aprovação final da versão do artigo a ser publicada. Andrade ACS, participou da concepção e planejamento do estudo; análise e interpretação dos dados; redação e revisão do artigo; aprovação final da versão do artigo a ser publicada.

\section{Agradecimentos}

Os autores agradecem ao Programa Institucional de Voluntariado de Iniciação Científica para alunos da graduação (VIC) e Programa Institucional de Bolsas de Iniciação Científica para alunos da graduação (PIBIC) da Universidade Federal de Mato Grosso - UFMT.

\section{Referências}

1. Cohen DA,McKenzie TL, Sehgal A, Williamson S, Golinelli D, Lurie N. Contribution of public parks to physical activity. Am J Public Health. 2007;97(3):509-14.

2. Florindo AA, Barrozo LV, Cabral-Miranda W, Rodrigues EQ, Turrell G, Goldbaum M, et al. Public open spaces and leisure-time walking in Brazilian adults. Int J Environ Res Public Health. 2017;14(6):553.

3. Hino AAF, Rech CR, Gonçalves PB, Reis RS. Acessibilidade a espaços públicos de lazer e atividade física em adultos de Curitiba, Paraná, Brasil. Cad Saúde Pública. 2019;35(12):e00020719.

4. Fermino RC, Reis RS, Cassou ACN. Fatores individuais e ambientais associados ao uso de parques e praças por adultos de Curitiba-PR, Brasil. Rev Bras Cineantropom Desempenho Hum. 2012;14(4):377-89.

5. Silva IJO, Alexandre MG, Ravagnani FCP, Silva JVP, Coelho-Ravagnani CF. Atividade física: espaços e condições ambientais para sua prática em uma capital brasileira. R Bras Ci e Mov. 2014;22(3):53-62.

6. McCormack GR, Rock M, Toohey AM, Hignell D. Characteristics of urban parks associated with park use and physical activity: A review of qualitative research. Health Place. 2010;16(4):712-26.

7. Fermino RC, Reis RS. Variáveis individuais, ambientais e sociais associadas com o uso de espaços públicos abertos para a prática de atividade física: uma revisão sistemática. Rev Bras Ativ Fís Saúde. 2013;18(5):523-35.

8. Silva MC, Silva AB, Amorim TEC. Condições de espaços públicos destinados à prática de atividades físicas na cidade de Pelotas/RS/Brasil. Rev Bras Ativ Fis Saúde. 2012;17(1):28-32.

9. Manta SW, Reis RS, Benedetti TRB, Rech CR. Public open spaces and physical activity: disparities of resources in Florianópolis. Rev Saúde Pública. 2019;53:112.

10. Silva EAPC, Silva PPC, Oliveira LS, Santos ARM, Rechia S, Freitas CMSM. Percepção da qualidade do ambiente e vivências em espaços públicos de lazer. Rev Bras Cienc Esporte. 2016;38(3):251-58. 
11. Bedimo-Rung AL, Mowen AJ, Cohen DA. The significance of parks to physical activity and public health: a conceptual model. Am J Prev Med. 2005;28(2):159-68.

12. Bauman AE, Reis RS, Sallis JF, Wells JC, Loos RJF, Martin BW. Correlates of physical activity: why are some people physically active and others not? Lancet. 2012;380:258-71.

13. Boclin KLS, Faerstein E, De Leon ACMP. Características contextuais de vizinhança e atividade física de lazer: Estudo Pró-Saúde. Rev Saúde Pública. 2014;48(2):249-57.

14. Sallis JF, Bowles HR, Bauman A, Ainsworth BE, Bull FC, Craig CL, et al. Neighborhood environments and physical activity among adults in 11 countries. Am J Prev Med. 2009;36(6):484-90.

15. Moura R, Oliveira S, Pêgo B. Escalas da urbanização brasileira. Brasília: Instituto de Pesquisa Econômica Aplicada - Ipea, 2018.

16. Pereira RHM, Braga CKV, Serra B, Nadalin VG. Desigualdades socioespaciais de acesso a oportunidades nas cidades brasileiras. Brasília: Instituto de Pesquisa Econômica Aplicada - Ipea, 2019.

17. Observatório das Metrópoles. IBEU MUNICIPAL: Índice de Bem-Estar Urbano dos municípios brasileiros. 2016. [citado em 2019 nov 6]. Disponível em: http://observatoriodasmetropoles.net.br/download/ ibeumunicipal_2016.pdf\&gt.

18. Leite C, Acosta C, Herling T, Barrozo L, Saldiva P. Indicadores de desigualdade para financiamento urbano de cidades saudáveis. Estud Av. 2019;33(97):37-60.

19. Figueiredo GLA, Martins CHG, Damasceno JL, Castro GGD, Mainegra AB, Akerman, M. Direito à cidade, direito à saúde: quais interconexões? Ciênc Saúde Colet. 2017;22(12):3821-830.

20. Ribeiro MCSA, Barata RB. Saúde: vulnerabilidade social, vizinhança e atividade física. Cad Metrópole. 2016;18(36):401-20.

21. Szwarcwald CL, Malta DC, Pereira CA, Vieira MLFP, Conde WL, Souza Júnior PRB, et al. Pesquisa Nacional de Saúde no Brasil: concepção e metodologia de aplicação. Cien Saude Colet. 2014;19(2):333-42.
22. Observatório das Metrópoles. Dados. 2014. [citado em 2019 nov 6]. Disponível em: https://ibeu. observatoriodasmetropoles.net.br/dados/

23. Callegari-Jacques SM. Bioestatística: princípios e aplicações. Porto Alegre: Artmed; 2003.

24. SEMEIA. Parques do Brasil: Percepções da População. 2020. [citado em 2020 mai 10]. Disponível em: http://www.semeia. org.br/arquivos/2020_PercepcoesdaPopulacao_V6.pdf

25. Sakata FG, Gonçalves FM. Um novo conceito para parque urbano no Brasil do século XXI. Paisag Ambiente: Ensaios. 2019;30(43):e155785.

26. Fermino RC, Hallal PC, Reis RS. Frequência de uso de parques e prática de atividades físicas em adultos de Curitiba, Brasil. Rev Bras Med Esporte. 2017;23(4):264-70.

27. Malta DC, Andrade SSA, Santos MAS, Rodrigues GBA, Mielke GI. Tendências dos indicadores de atividade física em adultos: Conjunto de capitais do Brasil 2006-2013. Rev Bras Ativ Fis Saúde. 2015;20(2):141-51.

28. IPEA. Retrato das Desigualdades de Gênero e Raça - 1995 a 2015. 2015. [citado em 2020 jun 15]. Disponível em: https:// www.ipea.gov.br/portal/images/stories/PDFs/170306_ retrato_das_desigualdades_de_genero_raca.pdf

29. Lee RE, Mama SK, Adamus-Leach HJ, Soltero EG. Contribution of neighborhood income and access to quality physical activity resources to physical activity in ethnic minority women over time. Am J Health Promot. 2015;29(4):210-16.

30. Salvo D, Sarmiento OL, Reis RS, Hino AAF, Bolivar MA, Lemoine PD, et al. Where Latin Americans are physically active, and why does it matter? Findings from the IPENadult study in Bogota, Colombia; Cuernavaca, Mexico; and Curitiba, Brazil. Prev Med. 2017;103:S27-S33.

Recebido: 01/09/2020

Aprovado: 23/04/2021

\section{Como citar este artigo:}

Agrizzi P, Dourado TEPS, Silva JI, Andrade ACS. Fatores associados ao conhecimento de locais públicos de esporte e lazer nas capitais brasileiras. Rev Bras Ativ Fis Saúde. 2021;26:e0201. DOI: 10.12820/rbafs.26e0201 